\title{
A Multicenter, Randomized, Controlled Trial for Assessing the Usefulness of Suppressing Thyroid Stimulating Hormone Target Levels after Thyroid Lobectomy in Low to Intermediate Risk Thyroid Cancer Patients (MASTER):
}

\section{A Study Protocol}

Eun Kyung Lee ${ }^{1, *}$, Yea Eun Kang ${ }^{2, *}$, Young Joo Park ${ }^{3}$, Bon Seok Koo ${ }^{4}, \mathrm{Ki}-$ Wook Chung ${ }^{5}$, Eu Jeong Ku $\mathrm{Ku}^{6}$, Ho-Ryun Won ${ }^{4,7}$, Won Sang Yoo ${ }^{8}$, Eonju Jeon ${ }^{9}$, Se Hyun Paek ${ }^{10}$, Yong Sang Lee ${ }^{11}$, Dong Mee Lim ${ }^{12}$, Yong Joon Suh ${ }^{13}$, Ha Kyoung Park ${ }^{14}$, Hyo-Jeong Kim ${ }^{15}$, Bo Hyun Kim ${ }^{16}$, Mijin Kim ${ }^{16}$, Sun Wook Kim ${ }^{17}$, Ka Hee Yi ${ }^{18}$, Sue K. Park ${ }^{19,20,21}$, Eun-Jae Jung ${ }^{22}$, June Young Choi ${ }^{23}$, Ja Seong Bae ${ }^{24}$, Joon Hwa Hong ${ }^{25}$, Kee-Hyun Nam ${ }^{26}$, Young Ki Lee ${ }^{1}$, Hyeong Won $\mathrm{Yu}^{23}$, Sujeong $\mathrm{Go}^{1}$, Young Mi Kang ${ }^{4}$, MASTER study group

${ }^{1}$ Center for Thyroid Cancer, National Cancer Center, Goyang; ${ }^{2}$ Department of Internal Medicine, Chungnam National University College of Medicine, Daejeon; ${ }^{3}$ Department of Internal Medicine, Seoul National University College of Medicine, Seoul; ${ }^{4}$ Department of Otorhinolaryngology-Head and Neck Surgery, Chungnam National University College of Medicine, Daejeon; ${ }^{5}$ Department of Surgery, Asan Medical Center, University of Ulsan College of Medicine, Seoul; ${ }^{6}$ Department of Internal Medicine, Chungbuk National University College of Medicine, Cheongju; ${ }^{7}$ Department of Otorhinolaryngology-Head and Neck Surgery, Chungnam National University Sejong Hospital, Sejong; ${ }^{8}$ Department of Internal Medicine, Dankook University College of Medicine, Cheonan; ${ }^{9}$ Department of Internal Medicine, Daegu Catholic University School of Medicine, Daegu; ${ }^{10}$ Department of Surgery, Ewha Womans University School of Medicine; ${ }^{11}$ Department of Surgery, Gangnam Severance Hospital, Yonsei University College of Medicine, Seoul; ${ }^{12}$ Department of Internal Medicine, Konyang University Hospital, Daejeon; ${ }^{13}$ Department of Breast and Endocrine Surgery, Hallym University Sacred Heart Hospital, Anyang; ${ }^{14}$ Department of Surgery, Inje University Busan Paik Hospital, Busan; ${ }^{15}$ Department of Internal Medicine, Nowon Eulji Medical Center, Eulji University, Seoul; ${ }^{16}$ Department of Internal Medicine, Pusan National University Hospital, Busan; ${ }^{17}$ Division of Endocrinology \& Metabolism, Department of Medicine, Thyroid Center, Samsung Medical Center, Sungkyunkwan University School of Medicine; ${ }^{18}$ Department of Internal Medicine, Seoul Metropolitan Government Seoul National University Boramae Medical Center; ${ }^{19}$ Department of Preventive Medicine, Seoul National University College of Medicine; ${ }^{20}$ Cancer Research Institute, Seoul National University; ${ }^{21}$ Convergence Graduate Program in Innovative Medical Science, ${ }^{22}$ Department of Otorhinolaryngology-Head and Neck Surgery, Seoul National University College of Medicine, Seoul; ${ }^{23}$ Department of Surgery, Seoul National University Bundang Hospital, Seongnam; ${ }^{24}$ Department of Surgery, Seoul St. Mary's Hospital, College of Medicine, The Catholic University of Korea, Seoul; ${ }^{25}$ Department of Internal Medicine, Daejeon Eulji Medical Center, Eulji University, Daejeon; ${ }^{26}$ Department of Surgery, Yonsei University College of Medicine, Seoul, Korea

Received: 14 December 2020, Revised: 17 February 2021 Accepted: 8 March 2021

Corresponding authors: Young Joo Park

Department of Internal Medicine, Seoul National University College of Medicine, 101 Daehak-ro, Jongno-gu, Seoul 03080, Korea

Tel: +82-2-2072-4183, Fax: +82-2-764-2199, E-mail: yjparkmd@snu.ac.kr

Bon Seok Koo

Department of Otorhinolaryngology-Head and Neck Surgery, Chungnam National University College of Medicine, 282 Munhwa-ro, Jung-gu, Daejeon 35015, Korea Tel: +82-42-280-7690, Fax:+82-42-253-4059, E-mail: bskoo515@cnu.ac.kr

\section{Copyright $\odot 2021$ Korean Endocrine Society}

This is an Open Access article distributed under the terms of the Creative Commons Attribution Non-Commercial License (https://creativecommons.org/ licenses/by-nc/4.0/) which permits unrestricted non-commercial use, distribution, and reproduction in any medium, provided the original work is properly cited.

*These authors contributed equally to this work. 
Background: Postoperative thyroid stimulating hormone (TSH) suppression therapy is recommended for patients with intermediate- and high-risk differentiated thyroid cancer to prevent the recurrence of thyroid cancer. With the recent increase in small thyroid cancer cases, the extent of resection during surgery has generally decreased. Therefore, questions have been raised about the efficacy and long-term side effects of TSH suppression therapy in patients who have undergone a lobectomy.

Methods: This is a multicenter, prospective, randomized, controlled clinical trial in which 2,986 patients with papillary thyroid cancer are randomized into a high-TSH group (intervention) and a low-TSH group (control) after having undergone a lobectomy. The principle of treatment includes a TSH-lowering regimen aimed at TSH levels between 0.3 and $1.99 \mu \mathrm{IU} / \mathrm{mL}$ in the low-TSH group. The high-TSH group targets TSH levels between 2.0 and $7.99 \mu \mathrm{IU} / \mathrm{mL}$. The dose of levothyroxine will be adjusted at each visit to maintain the target TSH level. The primary outcome is recurrence-free survival, as assessed by neck ultrasound every 6 to 12 months. Secondary endpoints include disease-free survival, overall survival, success rate in reaching the TSH target range, the proportion of patients with major cardiovascular diseases or bone metabolic disease, the quality of life, and medical costs. The followup period is 5 years.

Conclusion: The results of this trial will contribute to establishing the optimal indication for TSH suppression therapy in low-risk papillary thyroid cancer patients by evaluating the benefit and harm of lowering TSH levels in terms of recurrence, metabolic complications, costs, and quality of life.

Keywords: Thyroid neoplasms; Thyrotropin; Thyroxine; Recurrence; Thyroidectomy

\section{INTRODUCTION}

The incidence of thyroid cancer has been growing continuously, especially showing marked increments in the proportion of papillary thyroid microcarcinoma among all cases [1]. Recent guidelines recommend less surgery for the treatment of small thyroid cancers; accordingly, the popularity of a lobectomy has been increasing; a lobectomy was performed in $60.5 \%$ of cases involving 0.5 to $1 \mathrm{~cm}$ sized thyroid cancer in 2009 , whereas the rate was $81.2 \%$ in 2014 [2].

Thyroid stimulating hormone (TSH) suppression therapy is a mainstay in the management of differentiated thyroid cancer after surgery [3-5]. Given that TSH receptors are expressed in the membranes of normal thyrocytes as well as in thyroid cancer cells, lower levels of TSH suppress both the proliferation of thyroid cells and cancer growth [6]. One systematic review of ten articles showed that TSH suppression therapy reduces the recurrence of thyroid cancer (relative risk, 0.75 ; $95 \%$ confidence interval [CI], 0.60 to 0.88 ) [7]. However, only a few results focused on patients with a low risk of thyroid cancer who underwent only a lobectomy. One retrospective study showed that there was no difference in recurrence levels between the patients of TSH $<2 \mu \mathrm{IU} / \mathrm{mL}$ and those of TSH $\geq 2 \mu \mathrm{IU} / \mathrm{mL}$ [8]. Another retrospective study analyzed 783 lobectomy patients over a course of 8.6 years and suggested that the recurrence rate of thyroid cancer in patients of TSH $<2 \mu \mathrm{IU} / \mathrm{mL}$ did not differ from that of patients of TSH $\geq 2 \mu \mathrm{IU} / \mathrm{mL}$ [9].
Lower serum TSH concentration levels have led to some concern about the risk of several metabolic complications. Cardiovascular disease is the most common cause of non-cancer deaths in those with thyroid cancer [10], especially in patients with serum TSH concentrations of less than $0.02 \mu \mathrm{IU} / \mathrm{mL}$, who show an increased risk of cardiovascular death compared to those whose concentrations exceed $0.2 \mu \mathrm{IU} / \mathrm{mL}$ [11]. TSH suppression is also associated with bone metabolic disease. Lee et al. [12] showed that long-term TSH suppression reduced bone mineral density levels in post-menopausal women after a thyroidectomy. Wang et al. [13] showed that TSH suppression therapy was associated with a greater risk of osteoporosis but failed to reduce cancer recurrence. Furthermore, approximately $75 \%$ to $90 \%$ of TSH suppressed patients required levothyroxine (LT4) $[9,14]$, resulting in a poor quality of life (QoL) [15-17].

Therefore, we hypothesize that less TSH suppression therapy may not be linked to an increase in cancer recurrence in patients who have undergone a lobectomy for the treatment of papillary thyroid cancer (PTC) with fewer metabolic complications. We will also assess the QoL and medical costs in both groups. We will undertake a 5 -year multi-center prospective randomized controlled clinical trial to compare relapse-free survival rates, incidences of cardiovascular events and osteoporosis, the QoL, and medical costs between a high-TSH group (intervention) and a low-TSH group (control). 


\section{METHODS}

\section{Study design and setting}

This is a multicenter, randomized, parallel-group, open-label, standard treatment-controlled trial (Fig. 1). The study protocol was designed following the Standard Protocol Item Recommendations for Interventional Trials (SPIRIT) of 2013 [18]. Patients who meet the eligibility criteria will be recruited from 21 referral hospitals in Korea. This trial will be conducted in accordance with the principles of the Declaration of Helsinki and the Good Clinical Practice guidelines of the International Conference on Harmonization.

The study protocol was approved by the ethics committees of the following participating institutions: Seoul National University Hospital (H-1912-012-1084), Nowon Eulji Medical Center (EMCS 2020-04-020), Asan Medical Center (2020-0529), Inje University Busan Paik Hospital (19-0227), Chungnam National University Hospital (CNUH2020-03-091), Chungnam National University Sejong Hospital (CNUSH2020-11-018), Konyang University Hospital (KYUH2020-05-015), Daegu Catholic University Hospital (CR-20-125-L), Samsung Medical Center (SMC 2020-09-184), National Cancer Center (NCC 2020-0109), Seoul Metropolitan Government Seoul National University Boramae Medical Center (10-2020-035), Severance Hospital (2020-0202001), Chungbuk National University Hospital (2019-11-012), Eulji University Hospital (2020-02-005), Dankook University Hospital (2019-11-033), Seoul National University Bundang Hospital (B-2006/618-403), Ewha Womans University Seoul
Medical Center (SEUMC 2019-11-035), Hallym University Sacred Heart Hospital (HALLYM 2019-05-022), Pusan National University Hospital (H-2006-023-091), Seoul St. Mary's Hospital (KC20EIDT0476), and the Gangnam Severance Hospital (2019-0794-001). The protocol of this study was registered at the Clinical Research Information Service (CRIS) of the Korea Centers for Disease Control and Prevention (KCT0004989) in May of 2020.

Participants are to be recruited during outpatient clinic sessions by their physicians from the abovementioned medical centers. We do not use any advertisements as part of additional recruitment efforts.

\section{Patients' eligibility criteria}

Thyroid cancer patients must meet the inclusion and exclusion criteria before informed consent can be obtained. The inclusion criteria are as follows: (1) age between 19 and 79 years old (male or non-pregnant female); (2) PTC patients who underwent a lobectomy within three weeks as part of their treatment for PTC; (3) tumor size of $\leq 4 \mathrm{~cm}$ with no or minimal extrathyroidal extension, clinical stage N0 or N1a according to American Joint Committee on Cancer (AJCC) tumor, node, metastasis (TNM) stage 8th Edition Cancer Staging [19]; and (4) no nodule or any nodules in remained lobes showing benign or low-suspicion ultrasonographic findings (Korean Thyroid Imaging Reporting and Data System [K-TIRADS] Class 1-3 [20]) with Bethesda system category I-III by fine needle aspiration (FNA) [21].

The exclusion criteria for the participants are as follows: (1)

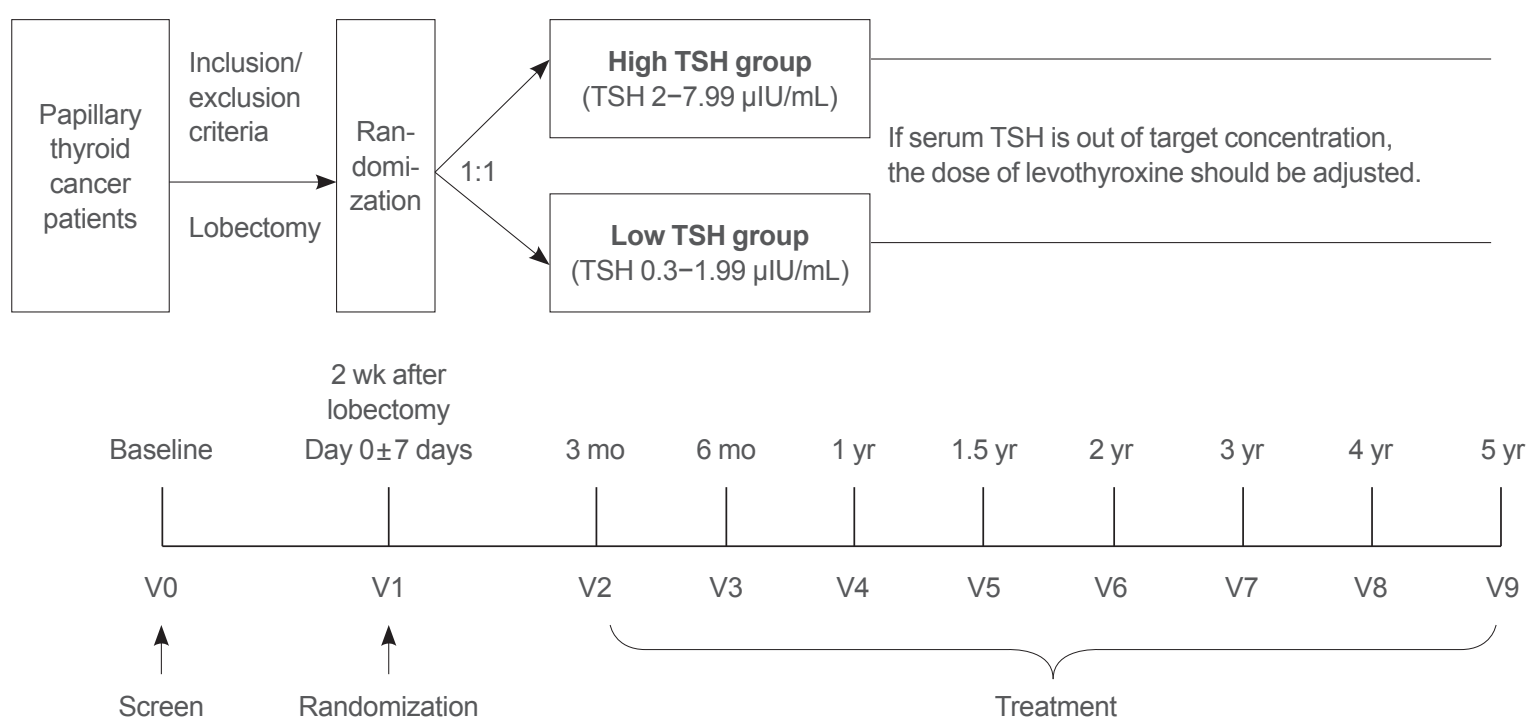

Fig. 1. Study flow chart. TSH, thyroid stimulating hormone. 
patients with an advanced stage of pT4, pN1b, or M1; (2) aggressive histological variants of PTC (poorly differentiated, anaplastic, or diffuse sclerosing types); (3) histological types other than PTC (follicular, anaplastic, or medullary thyroid cancer); (4) necessary for completion thyroidectomy; (5) incomplete tumor resection (R1/R2); (6) any incidence of follicular neoplasm, suspicious for malignancy or malignancy (Bethesda category IV, V, or VI) according to FNA in the remaining lobes or incomplete evaluation; (7) active hyperthyroidism; or (8) any other clinical conditions that in the opinion of the investigator would make the subject unsuitable for the study.

\section{Intervention and comparator}

The high-TSH group (intervention group) will target serum TSH levels between 2.0 and $7.99 \mu \mathrm{IU} / \mathrm{mL}$, while the low-TSH group (comparison group) target serum TSH levels between 0.3 and $1.99 \mu \mathrm{IU} / \mathrm{mL}$. If the serum TSH level is out of the target range, the dose of LT4 will be adjusted at every visit or the TSH level will be rechecked according to the patient's individual status of compliance, medication method, and changes in other medication etc. LT4 should be administered orally every day 1 hour or more before a meal or 3 hours or more after a meal. The dose adjustment will be determined by each investigator based on the dose adjustment protocol (Table 1). Compliance with medication is defined as the consumption of at least $80 \%$ of the medication.

\section{Randomization}

PTC patients who meet the eligibility criteria will be randomized at a 1:1 ratio into the high-TSH group or the low-TSH group, stratified for sex and institute. A stratified randomization method developed by the Medical Research Collaborating Center of Seoul National University Hospital will be used.

$\begin{aligned} & \text { Table } 1 . \text { Dose Adjustment Protocol of Levothyroxine Based on } \\
& \text { Serum TSH Levels }\end{aligned}$
\begin{tabular}{lcc}
\hline $\begin{array}{l}\text { Serum TSH level, } \\
\mu \mathrm{IU} / \mathrm{mL}\end{array}$ & $\begin{array}{l}\text { High TSH group } \\
\text { (target } 2.0-7.99 \mu \mathrm{IU} / \mathrm{mL})\end{array}$ & $\begin{array}{c}\text { Low TSH group } \\
\text { (target } 0.3-1.99 \mu \mathrm{IU} / \mathrm{mL})\end{array}$ \\
\hline$<0.01$ & -25 to $50 \mu \mathrm{g} /$ day & $-25 \mu \mathrm{g} /$ day \\
$0.01-0.29$ & -12.5 to $25 \mu \mathrm{g} /$ day & $-12.5 \mu \mathrm{g} /$ day \\
$0.3-1.99$ & $-12.5 \mu \mathrm{g} /$ day & No change \\
$2.0-7.99$ & No change & $12.5 \mu \mathrm{g} /$ day \\
$8.0-19.9$ & $12.5 \mu \mathrm{g} /$ day & $25 \mu \mathrm{g} /$ day \\
$\geq 20$ & $25 \mu \mathrm{g} /$ day & 37.5 to $50 \mu \mathrm{g} /$ day \\
\hline TSH, thyroid stimulating hormone.
\end{tabular}

\section{Outcomes}

The primary endpoint is the relapse-free survival of PTC for 5 years, as assessed by neck ultrasound (US) every 6 to 12 months. If recurrence is suspected, pathological confirmation is mandatory. Secondary endpoints are disease-free survival, disease-specific survival, overall survival, a success rate to reach the TSH target range, the proportion of patients with major cardiovascular events (MACE), atrial fibrillation, or bone metabolic disease (e.g., osteoporosis, osteopenia, fracture), QoL, and medical costs. The exploratory endpoints are searching for risk factors associated with recurrence and the development of complications and obtaining blood or tissue samples for storage for examinations of biomarkers.

\section{Sample size}

The sample size in this study was calculated by means of a noninferiority test under the following conditions: the proportion of subjects who experience an event during the study is 0.05 for the comparison group and 0.05 for the treatment group under the assumption that the risk ratio between two arms is constant throughout the study periods; actual ratio $=1.0$ and non-inferiority hazard ratio $(\mathrm{HR})=1.67$, alpha error $=0.025$, beta error $=0.2$, and sample allocation ratio $=1.0$ [22]. The calculated sample size was 1,194 for each arm (a total 2,388). Considering a dropout rate of $20 \%$, in total, 2,986 patients must be enrolled.

\section{Repeated visit, follow-up, and data collection}

The following data will be collected for both groups: socioanthropometric characteristics, body mass index, smoking and alcohol habits, underlying morbidities, previous and newly diagnosed cardiovascular diseases and osteoporosis, medications during the follow-up of the study period and laboratory test.

After randomization, participants will be requested to visit an outpatient clinic every 3 months during the first 6 months (V2$\mathrm{V} 3$ ), every 6 months for 2 years (V4-V6), and then annually thereafter (V7-V9). At each visit, a thyroid function test, neck US, a thyroglobulin ( $\mathrm{Tg}$ ) assessment, a Tg antibody (Ab) test, a questionnaire on QoL, and an event occurrence assessment, i.e., admission for MACE or fracture, will be conducted. Symptoms associated with hyperthyroidism, cardiovascular disease and fracture will be interviewed by a clinician. When participants need to visit the hospital to receive medicine for metabolic complications or due to any specific symptoms, they will be asked to refer to a specialist. Bone mineral density levels, a body impedance analysis, hand grip strength and electrocardiography will be assessed every 2 years optionally.

www.e-enm.org

577 


\section{Data analysis plan}

The population of the intention-to-treat full analysis set (FAS) is defined as participants who have completed randomization and been assessed as reaching a primary endpoint at least once. The per-protocol population (PP) analysis includes only those participants who have strictly followed the intervention protocol (to obtain the target TSH level) and have completed the follow-up. The population comprising the safety analysis set is defined as participants who have completed randomization.

The statistical analyses for the primary endpoint will be performed using the PP and FAS population, and non-inferiority should be shown for both the PP and FAS populations. A onesided test will be conducted, and $P$ values of $<0.025$ will be considered statistically significant. Other endpoints will be performed with the FAS via a two-sided test, and $P$ values of $<0.05$ will be considered statistically significant (unless otherwise specified).

A Kaplan-Meier analysis will be used as a survival analysis and to calculate the survival rate and $95 \% \mathrm{CI}$. The Cox proportional-hazards model will be used to compare the two groups and to calculate the HR and 95\% CI. The calculated HR $<1.67$ (non-inferiority) will be interpreted to confirm non-inferiority of the intervention group (high TSH) relative to the comparison group (low TSH). The stratified Cox proportional-hazards model will be used to calculate the adjusted HR stratifying for sex and institute.

Numeric variables will be presented as the mean \pm standard deviation or median (minimum-maximum or interquartile range), and categorical variables will be presented as the number of cases (percentage). The data will be analyzed by an independent samples $t$ test, the Wilcoxon rank sum test, a chi-square test, a continuity-corrected chi-square test or Fisher's exact test. The events of osteoporosis and MACE, the medical costs, and mortality caused by thyroid cancer will be compared between the two groups. Subgroup analyses using factors such as age, tumor stage, and surgical method will be conducted.

\section{Trial status}

This trial was registered at the CRIS on May 6, 2020 (https:// cris.nih.go.kr/cris/search/search_result_st01.jsp?seq=16437). The recruitment started on May 14, 2020 and is ongoing. The current protocol version is 1.13 from April 27, 2020.

\section{Data sharing plan}

When the trial is completed, we plan to disseminate the results of this trial at national and international meetings that target im- portant audiences. Results will be prepared and edited into manuscript(s) for peer-review publication. The raw data from the trial will be available by request with privacy protection after the approval of the relevant data management committee.

\section{DISCUSSION}

This study protocol describes the design and methods of the 'Multicenter Randomized Controlled Study for Assessing the Usefulness of Suppressing Thyroid Stimulating Hormone Target Levels after Thyroid lobEctomy in Low to Intermediate Risk Thyroid Cancer Patients (MASTER)' study. The MASTER study is designed to compare relapse-free survival, the incidence of cardiovascular events and osteoporosis, the QoL, and medical costs between the high-TSH group (intervention) and the low-TSH group (control). In this study, we focus on not only clinical outcomes of exposure to high serum TSH levels in PTC patients but also the changes in the QoL and cost difference between the two choices of low TSH and high TSH levels.

In recent years, minimal treatment has been emphasized to improve the QoL and reduce complications for thyroid cancer patients. Nevertheless, for serum TSH levels it is recommended to keep it below $2 \mu \mathrm{IU} / \mathrm{mL}$, and lower it further below that, especially in patients at high risk of recurrence. However, it is difficult to keep serum TSH levels at target levels in a clinical practice setting. One retrospective study found that $441(80 \%)$ of 1,528 patients who underwent lobectomy required LT4 to lower TSH to less than $2.0 \mu \mathrm{IU} / \mathrm{mL}$, and $9.8 \%$ of patients, despite LT4 treatment, had an average TSH higher than $4.5 \mu \mathrm{IU} /$ $\mathrm{mL}$ [23]. Fortunately, LT4 treatment with elevated TSH did not affect relapse-free survival (HR, 1.74; 95\% CI, 0.50 to 5.99; $P=0.38)$. Therefore, we have assumed that strict maintenance of serum TSH level is not mandatory to bring good outcomes for thyroid cancer patients.

Higher serum TSH level as well as lower TSH level increases the risk of cardiovascular events and cardiovascular deaths, even in the range of asymptomatic hypothyroidism. The HRs for coronary heart disease was 1.17 (95\% CI, 0.96 to 1.43) for a TSH level of 7.0 to $9.9 \mathrm{mIU} / \mathrm{L}$ and 1.89 (95\% CI, 1.28 to 2.80$)$ for a TSH of 10.0 to $19.9 \mu \mathrm{IU} / \mathrm{mL}$ [24] and for heart failure 1.86 (95\% CI, 1.27 to 2.72 ) for TSH of 10.0 to $19.9 \mu \mathrm{IU} / \mathrm{mL}$ [25], respectively. Even in Koreans, subclinical hypothyroidism in the range of TSH $>6.57 \mu \mathrm{IU} / \mathrm{mL}$ had significantly higher cardiovascular risk and risk of all cause deaths [26]. Therefore, early treatment for asymptomatic hypothyroidism of TSH $\geq 8 \mu \mathrm{IU} /$ $\mathrm{mL}$ should be considered if subjects under 65 years of age have 
symptoms or have cardiac risk factors [27-29]. From these findings, the upper limit of TSH was determined as $7.99 \mu \mathrm{IU} / \mathrm{mL}$ to avoid enhancing cardiovascular risk in the high TSH group.

There are several strengths of this study. First, this is a prospective cohort study which could assist in determining causal associations between serum TSH levels and PTC recurrence in lobectomized PTC patients. Second, the face-to-face interview method may be especially useful for obtaining accurate information, especially when done by an experienced nurse practitioner. Third, the study recruited patients from multiple centers-21 centers in Korea. This will help to decrease selection bias according to hospital characteristics. Fourth, the MASTER study group is multidisciplinary and includes experts in endocrinology and general surgery or otolaryngology surgery. Fifth, random assignment is helpful to avoid biased results. The major limitation of the study is the absence of double-blind intervention, as the study drug is not manufactured for this study but is a commercially available drug in this case.

The MASTER study will contribute to establishing optimal indications for TSH suppression therapy in low-risk PTC patients by elucidating the benefits of TSH, i.e., lowering cancer recurrences and its detrimental effects, i.e., metabolic complications, reduced life quality, and higher costs. This will not only improve our understanding of the biological effects of thyroid hormones in thyroid cancer patients but will also assist in efforts to improve tailored management policies for PTC patients.

\section{CONFLICTS OF INTEREST}

No potential conflict of interest relevant to this article was reported.

\section{ACKNOWLEDGMENTS}

The following collaborators from the MASTER study group contributed to the recruitment and care of study participants and collected data.

Asan Medical Center: Ki-Wook Chung, Cheong-Sil Rah; Chungbuk National University: Eu Jeong Ku, Jin-Woo Park, Su Yeon Kim; Chungnam National University: Bon Seok Koo, Yea Eun Kang, Minho Shong, Hyon-Seung Yi, Ju Hee Lee, Jae Won Chang, Young Mi Kang, Minji Kim; Chungnam National University Sejong Hospital : Ho-Ryun Won, Kyong Hye Joung, Ji Min Kim, Younju Lee; Dankook University: Won Sang Yoo, Hyun Kyung Chung, Seung Hoon Woo; Daegu Catholic University: Eonju Jeon, Young Ju Jeong, Sung-Woo Kim; Ewha
Womans University School of Medicine: Se Hyun Paek, JongKyu Kim; Gangnam Severance Hospital, Yonsei University College of Medicine: Yong Sang Lee, Soo Young Kim, and Hang-Seok Chang; Hallym University Sacred Heart Hospital: Yong Joon Suh, Seong Jin Lee, Yun Kyung Cho; Inje University Busan Paik Hospital: Ha Kyoung Park, Tae Kwun Ha; Konyang University Hospital: Dong Mee Lim; National Cancer Center: Eun Kyung Lee, Yuh-Seog Jung, Junsun Ryu, Changhwan Ryu, You Jin Lee, Yul Hwangbo, Young Ki Lee, Sujeong Go; Nowon Eulji Medical Center, Eulji University: Hyo-Jeong Kim, Kyong Yeun Jung, Ah Ra Jung; Pusan National University Hospital: Bo Hyun Kim, Mijin Kim, Byung-Joo Lee, Sung-Chan Shin; Samsung Medical Center: Sun Wook Kim; Seoul Metropolitan Government Seoul National University Boramae Medical Center: Ka Hee Yi, Young Jun Chai; Seoul National University: Young Joo Park, Sue K. Park, Eun-Jae Jung, Su-Jin Kim, Soon Hyun Ahn, Kyu Eun Lee, Sun Wook Cho, Yoo Hyung Kim; Seoul National University Bundang Hospital: June Young Choi, Hyeong Won Yu, Jae Hoon Moon, Chang Ho Ahn; Seoul St. Mary's Hospital: Ja Seong Bae, Chae Ho Jung, Dong Joon Lim, Jeong Soo Kim, Jung Hoon Ha, Kwangsoon Kim; Daejeon Eulji Medical Center, Eulji University: Joon Hwa Hong; Yonsei University College of Medicine: Kee-Hyun Nam, Jin Kyong Kim, Dong Gyu Kim.

This research was supported by a grant of the Patient-Centered Clinical Research Coordinating Center funded by the Ministry of Health \& Welfare, Republic of Korea (grant number: HI19C0481, HC19C0103).

The authors acknowledge the support of the Medical Research Collaborating Center of Seoul National University Hospital for their comments and guidance in developing a protocol.

\section{AUTHOR CONTRIBUTIONS}

Conception or design: E.K.L., Y.E.K., Y.J.P., B.S.K., B.H.K., J.Y.C., E.J.K. Acquisition, analysis, or interpretation of data: E.K.L., Y.E.K., Y.J.P., B.S.K., K.W.C., E.J.K., H.R.W., W.S.Y., E.J., S.H.P., Y.S.L., D.M.L., Y.J.S., H.K.P., H.J.K., B.H.K., M.K., S.W.K., K.H.Y., S.K.P., E.J.J., J.Y.C., J.S.B., J.H.H., K.H.N., Y.K.L., H.W.Y., S.G., Y.M.K. Drafting the work or revising: E.K.L., Y.E.K., Y.J.P., B.S.K. Final approval of the manuscript: E.K.L., Y.E.K., Y.J.P., B.S.K., K.W.C., E.J.K., H.R.W., W.S.Y., E.J., S.H.P., Y.S.L., D.M.L., Y.J.S., H.K.P., H.J.K., B.H.K., M.K., S.W.K., K.H.Y., S.K.P., E.J.J., J.Y.C., J.S.B., J.H.H., K.H.N., Y.K.L., H.W.Y., S.G., Y.M.K. 


\section{ORCID}

Eun Kyung Lee https://orcid.org/0000-0003-0098-0873

Yea Eun Kang https://orcid.org/0000-0002-2012-3716

Young Joo Park https://orcid.org/0000-0002-3671-6364

Bon Seok Koo https://orcid.org/0000-0002-5928-0006

\section{REFERENCES}

1. Kitahara CM, Sosa JA. The changing incidence of thyroid cancer. Nat Rev Endocrinol 2016;12:646-53.

2. Lee YS, Chang HS, Park CS. Changing trends in the management of well-differentiated thyroid carcinoma in Korea. Endocr J 2016;63:515-21.

3. Haugen BR, Alexander EK, Bible KC, Doherty GM, Mandel SJ, Nikiforov YE, et al. 2015 American Thyroid Association management guidelines for adult patients with thyroid nodules and differentiated thyroid cancer: the American Thyroid Association guidelines task force on thyroid nodules and differentiated thyroid cancer. Thyroid 2016;26:1133.

4. Yi KH, Lee EK, Kang HC, Koh Y, Kim SW, Kim IJ, et al. 2016 Revised Korean Thyroid Association management guidelines for patients with thyroid nodules and thyroid cancer. Int J Thyroidol 2016;9:59-126.

5. Simpson WJ, Panzarella T, Carruthers JS, Gospodarowicz MK, Sutcliffe SB. Papillary and follicular thyroid cancer: impact of treatment in 1578 patients. Int J Radiat Oncol Biol Phys 1988;14:1063-75.

6. Carayon P, Thomas-Morvan C, Castanas E, Tubiana M. Human thyroid cancer: membrane thyrotropin binding and adenylate cyclase activity. J Clin Endocrinol Metab 1980;51: 915-20.

7. McGriff NJ, Csako G, Gourgiotis L, Guthrie LC, Pucino F, Sarlis NJ. Effects of thyroid hormone suppression therapy on adverse clinical outcomes in thyroid cancer. Ann Med 2002;34:554-64.

8. Lamartina L, Montesano T, Falcone R, Biffoni M, Grani G, Maranghi M, et al. Is it worth suppressing TSH in low- and intermediate-risk papillary thyroid cancer patients before the first disease assessment? Endocr Pract 2019;25:165-9.

9. Park S, Kim WG, Han M, Jeon MJ, Kwon H, Kim M, et al. Thyrotropin suppressive therapy for low-risk small thyroid cancer: a propensity score-matched cohort study. Thyroid 2017;27:1164-70.

10. Lee YK, Hong N, Park SH, Shin DY, Lee CR, Kang SW, et al. The relationship of comorbidities to mortality and cause of death in patients with differentiated thyroid carcinoma. Sci Rep 2019;9:11435.

11. Klein Hesselink EN, Klein Hesselink MS, de Bock GH, Gansevoort RT, Bakker SJ, Vredeveld EJ, et al. Long-term cardiovascular mortality in patients with differentiated thyroid carcinoma: an observational study. J Clin Oncol 2013; 31:4046-53.

12. Lee Y, Yoon BH, Lee S, Chung YK, Lee YK. Risk of osteoporotic fractures after thyroid-stimulating hormone suppression therapy in patients with thyroid cancer. J Bone Metab 2019;26:45-50.

13. Wang LY, Smith AW, Palmer FL, Tuttle RM, Mahrous A, Nixon IJ, et al. Thyrotropin suppression increases the risk of osteoporosis without decreasing recurrence in ATA low- and intermediate-risk patients with differentiated thyroid carcinoma. Thyroid 2015;25:300-7.

14. Cox C, Bosley M, Southerland LB, Ahmadi S, Perkins J, Roman S, et al. Lobectomy for treatment of differentiated thyroid cancer: can patients avoid postoperative thyroid hormone supplementation and be compliant with the American Thyroid Association guidelines? Surgery 2018;163:75-80.

15. Hedman C, Djarv T, Strang P, Lundgren CI. Fear of recurrence and view of life affect health-related quality of life in patients with differentiated thyroid carcinoma: a prospective Swedish population-based study. Thyroid 2018 Oct 26 [Epub]. https://doi.org/10.1089/thy.2018.0388.

16. Vigario Pdos S, Chachamovitz DS, Teixeira Pde F, Rocque Mde L, Santos ML, Vaisman M. Exercise is associated with better quality of life in patients on TSH-suppressive therapy with levothyroxine for differentiated thyroid carcinoma. Arq Bras Endocrinol Metabol 2014;58:274-81.

17. Grani G, Ramundo V, Verrienti A, Sponziello M, Durante C. Thyroid hormone therapy in differentiated thyroid cancer. Endocrine 2019;66:43-50.

18. Chan AW, Tetzlaff JM, Altman DG, Laupacis A, Gotzsche PC, Krleza-Jeric K, et al. SPIRIT 2013 statement: defining standard protocol items for clinical trials. Ann Intern Med 2013;158:200-7.

19. Perrier ND, Brierley JD, Tuttle RM. Differentiated and anaplastic thyroid carcinoma: major changes in the American Joint Committee on Cancer eighth edition cancer staging manual. CA Cancer J Clin 2018;68:55-63.

20. Shin JH, Baek JH, Chung J, Ha EJ, Kim JH, Lee YH, et al. Ultrasonography diagnosis and imaging-based management of thyroid nodules: revised Korean Society of Thyroid Radi-

Copyright (C) 2021 Korean Endocrine Society 
ology consensus statement and recommendations. Korean J Radiol 2016;17:370-95.

21. Cibas ES, Ali SZ. The 2017 Bethesda system for reporting thyroid cytopathology. Thyroid 2017;27:1341-6.

22. Chow SC, Shao J, Wang H. Sample size calculations in clinical research. 2nd ed. Hoboken: CRC press; 2007.

23. Lee MC, Kim MJ, Choi HS, Cho SW, Lee GH, Park YJ, et al. Postoperative thyroid-stimulating hormone levels did not affect recurrence after thyroid lobectomy in patients with papillary thyroid cancer. Endocrinol Metab (Seoul) 2019;34: 150-7.

24. Rodondi N, den Elzen WP, Bauer DC, Cappola AR, Razvi S, Walsh JP, et al. Subclinical hypothyroidism and the risk of coronary heart disease and mortality. JAMA 2010;304: 1365-74.

25. Gencer B, Collet TH, Virgini V, Bauer DC, Gussekloo J, Cappola AR, et al. Subclinical thyroid dysfunction and the risk of heart failure events: an individual participant data analysis from 6 prospective cohorts. Circulation 2012;126: 1040-9.

26. Moon S, Kim MJ, Yu JM, Yoo HJ, Park YJ. Subclinical hypothyroidism and the risk of cardiovascular disease and allcause mortality: a meta-analysis of prospective cohort studies. Thyroid 2018;28:1101-10.

27. Pearce SH, Brabant G, Duntas LH, Monzani F, Peeters RP, Razvi S, et al. 2013 ETA guideline: management of subclinical hypothyroidism. Eur Thyroid J 2013;2:215-28.

28. Jonklaas J, Bianco AC, Bauer AJ, Burman KD, Cappola AR, Celi FS, et al. Guidelines for the treatment of hypothyroidism: prepared by the American thyroid association task force on thyroid hormone replacement. Thyroid 2014;24: 1670-751.

29. Biondi B, Cappola AR, Cooper DS. Subclinical hypothyroidism: a review. JAMA 2019;322:153-60. 